\title{
Presence of sandfly-borne phleboviruses of two antigenic complexes (Sandfly fever Naples virus and Sandfly fever Sicilian virus) in two different
} bio-geographical regions of Tunisia demonstrated by a microneutralisation-based seroprevalence study in dogs

Sonia Sakhria ${ }^{1 \dagger}$, Sulaf Alwassouf ${ }^{2,3+}$, Wasfi Fares ${ }^{1}$, Laurence Bichaud ${ }^{2,3}$, Khalil Dachraoui ${ }^{1}$, Cigdem Alkan ${ }^{2,3}$, Ziad Zoghlami ${ }^{1}$, Xavier de Lamballerie ${ }^{2,3}$, Elyes Zhioua ${ }^{1}$ and Remi N Charrel ${ }^{2,3^{*}}$

\begin{abstract}
Background: Sandfly-borne phleboviruses are present in North Africa where they can infect humans in regions where Leishmania infantum, the causative agent of zoonotic visceral leishmaniasis in the Western Mediterranean basin is present affecting both humans and dogs. We investigated the capacity of dogs to be used as sentinels for sandfly-borne phleboviruses as previously shown for leishmaniasis.

Findings: A total of 312 sera were collected from guard dogs in two different bioclimatic regions (governorates of Kairouan and Bizerte) of Tunisia where zoonotic visceral leishmaniasis has been reported. These sera were tested for the presence of neutralising antibodies against 3 phleboviruses: Toscana virus, Punique virus and Sicilian virus. In the governorate of Kairouan, seroprevalence rates of $7.5 \%, 43.5 \%$, and $38.1 \%$ were observed for Toscana, Punique and Sicilian virus, respectively. A high proportion of sera from the governorate of Bizerte were hemolyzed and showed high cytotoxicity for the cells and subsequently precluded detailed interpretation of this batch. However, validated results for 27 sera were in agreement with data observed in the governorate of Kairouan.
\end{abstract}

Conclusions: Toscana virus is present in the governorate of Kairouan but at a lower rate compared to Punique and Sicilian viruses. These three sandfly-borne phleboviruses can infect dogs. Direct detection and isolation of the viruses are now to be attempted in animals as well as in humans. Our findings showed that guard dogs are good sentinels for virus transmitted by sandflies and strongly suggested that the high seroprevalence rates observed in dogs merit further attention.

Keywords: Phleboviruses, Toscana virus, Sand flies, Dogs, Sentinels, Emerging, Mediterranean basin, Bunyaviridae

\footnotetext{
* Correspondence: remi.charrel@univ-amu.fr

${ }^{\dagger}$ Equal contributors

${ }^{2}$ Aix Marseille Université, IRD French Institute of Research for Development,

EHESP French School of Public Health, EPV UMR_D 190 "Emergence des

Pathologies Virales", 13385 Marseille, France

${ }^{3}$ IHU Méditerranée Infection, APHM Public Hospitals of Marseille, 13385

Marseille, France

Full list of author information is available at the end of the article
} 


\section{Background}

Recent studies have indicated that sandfly-borne phleboviruses (genus Phlebovirus, family Bunyaviridae) were not only geographically restricted to southern Europe, but were also present in North Africa [1-6]. At least, viruses of 3 different antigenic complexes are transmitted by sandflies in the Old World: Salehabad complex, Sandfly fever Sicilian complex and Sandfly fever Naples complex. Two phleboviruses of the Sandfly fever Naples complex were reported to be present in Tunisia, namely Toscana virus and Punique virus as assessed by virus isolation [2,7]. Another virus, provisionally named Utique virus that belongs to the Sandfly fever Sicilian complex was genetically detected in Tunisia but it has not been isolated yet [7]. A recent sero-epidemiological study conducted in the governorate of Bizerte (northern Tunisia) showed that both Toscana and Punique viruses could infect human populations although Toscana virus was much more prevalent than Punique virus [5]. In Tunisia, Toscana, Punique and Utique viruses were detected and/or isolated from Phlebotomus pernicious and Phlebotomus longicuspis, which are considered as possible vectors of phleboviruses [2,7]. Both sandfly species are also the main vectors of Leishmania infantum, etiologic agent of zoonotic visceral leishmaniasis (ZVL) in Tunisia [8-10].

Dogs are the main reservoir host of L. infantum and therefore they are used as sentinels to assess the risk of ZVL and other zoonotic vector-borne diseases [11]. Phlebotomus pernicious and P. longicuspis are widely distributed in Tunisia [12], and subsequently, we hypothesized a large distribution of sandfly-borne phleboviruses.

It is important to point out that the governorate of Kairouan is the most endemic foci for ZVL [10]; in addition approximately $40 \%$ of the rural human population living in the governorate of Bizerte possesses antibodies neutralizing Toscana virus (TOSV-NT-Ab) [5]. Therefore, human populations are exposed to sandflyborne diseases in both governorates.

\section{Findings}

The study took place in the governorate of Bizerte located in Northern Tunisia and in the governorate of Kairouan located in Central Tunisia corresponding to two different bio-geographical areas (Figure 1). To assess the circulation of sandfly-borne phleboviruses, dogs were used as sentinel. A retrospective study on dogs was undertaken in several districts of the governorates of Bizerte and Kairouan during the fall of 2013. Sampling was performed in five locations belonging to different bio-climatic zones varying from humid to arid (Sejnane: $36^{\circ} 56^{\prime} \mathrm{N}, 9^{\circ} 21^{\prime} \mathrm{E}$, humid; Mateur: 37 $03^{\prime} \mathrm{N}, 9^{\circ} 28^{\prime} \mathrm{E}$, Sub-humid; Borj Youssef; $36^{\circ} 56^{\prime} \mathrm{N}, 10^{\circ} 07^{\prime} \mathrm{E}$, semi-arid; Haffouz: $34^{\circ} 51 \mathrm{~N}$, $9^{\circ} 29^{\prime} \mathrm{E}$, arid; Bouhajla, 3524’ N, 956' E, arid) (Figure 1). The selected sites were restricted to previously surveyed areas characterized by the abundance of phlebotomine species of the subgenus Larroussius [2,10,12]. The typical setting of a house in endemic areas for ZVL includes the guard dog, attached for his entire life close to the house, and to the sheep shed and chicken henhouse. Houses are always surrounded by cactus to provide protection against trespassing and cactus peers are highly appreciated by villagers. This ecological setting offers suitable biotope for sandflies. Sheep sheds made usually with mud walls are breeding sites for sandflies (Zhioua, unpublished data). Flowers of the cactus are the only sugar source available around. Animals located in the peridomestic areas are the main source of blood meal including humans for sandflies. Dogs are the main source of L. infantum infection to sandflies. We organized door-to-door visits with a local veterinarian and a health worker who introduced the team to the local population. Information regarding age, sex, race was obtained after interviewing dog owners who gave their consent to be involved in the study. After filling out the questionnaire, each dog was examined clinically by the veterinarian and a 2-ml blood sample was collected by venepuncture of the forelimb. This study was performed following approval from the IACUC of Pasteur Institute of Tunis, Tunisia IPT/UESV/19/2010.

Dog sera were tested by using a microneutralisation assay performed comparatively with (i) two viruses belonging to the Sandfly fever Naples species (Toscana virus and Punique virus), (ii) and one virus belonging to the Sandfly fever Sicilian species (Sicilian virus) as described previously [5]. Neutralisation is the most discriminative serological assay that is well-adapted to differentiate the affinity of antibodies against different viruses. In addition, there is almost no cross-reaction.

From the governorates of Kairouan and Bizerte, 194 and 118 sera were collected from guard dogs, respectively. The virus microneutralisation (VNT) assay was performed in 96-well microtitre plates using Vero cells as previously described [5] with slight technical modifications. Briefly, twofold serial dilutions from 1:10 to 1:80 of a $50 \mu \mathrm{L}$-serum was mixed with an equal volume of virus culture titered at 1000 TCID50 into 96-well plates, providing two-fold final dilutions from 1:20 to $1: 160$.

For this study the 3 virus strains were (i) Toscana virus strain MRS2010-4319501 [13], (ii) Punique virus strain Tunisia2009T101 [7], (iii) Sandfly fever Sicilian virus strain Sabin [14]. The plate was incubated at $37^{\circ} \mathrm{C}$ for one hour, then a $100 \mu \mathrm{L}$ volume of Vero cells containing approximately $2.10^{4}$ cells in $5 \%$ foetal bovine serum was added to each well, and incubated at $37^{\circ} \mathrm{C}$ in presence of $5 \% \mathrm{CO}_{2}$. After 5 days, the microplates were read under an inverted microscope, and the presence or absence of cytopathic effect was noted. The titre (no neutralisation, neutralisation at 1:20, 1:40, 1:80 and 1:160) was recorded. The threshold for positivity was defined as 1:20 [5]. 


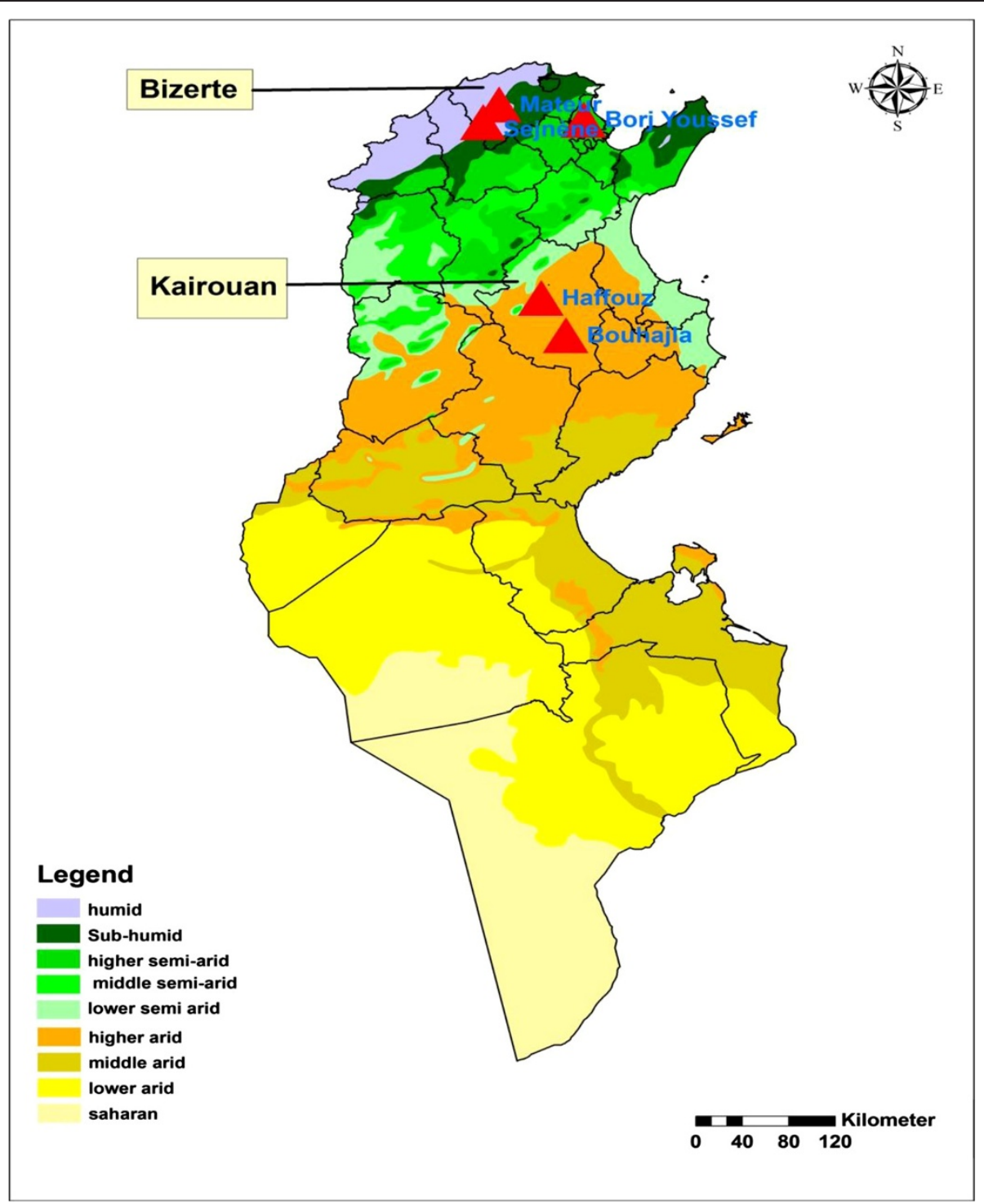

Figure 1 Sampling sites of sera from dogs.

The results are presented in Table 1. Of the 194 sera originating from Kairouan, 47 were cytotoxic for the cells, and therefore calculations were done on the basis of 147 sera. In the governorate of Bizerte, only 27 sera were considered as suitable for calculation because the proportion of sera showing cytotoxicity was much higher. Thus, the very low number precluded any statistical calculations.
Cytotoxicity was frequently observed with hemolyzed sera, resulting from suboptimal conditions of storage.

The results obtained from the analysis of 147 sera from Kairouan showed that the 3 viruses (or very closely related viruses) were circulating in the region. Seroprevalence rates of $43.5 \%$, and $38.1 \%$ were observed for Punique virus and Sicilian virus, respectively. In contrast, only $7.5 \%$ of

Table 1 Microneutralisation-based seroprevalence rates for Toscana, Punique and Sicilian viruses on 312 sera collected from guard dogs in Tunisia

\begin{tabular}{lllll}
\hline Tested sera & Cytotoxic for Vero cells & Toscana virus $\mathbf{n}(\%)$ & Punique virus $\mathbf{n}(\%)$ & Sicilian virus $\mathbf{n}(\%)$ \\
\hline Kairouan, $\mathbf{n}=\mathbf{1 9 4}$ & 47 & $11(7.5 \%)$ & $64(43.5 \%)$ & $56(38.1 \%)$ \\
Distribution of VNT titres $\mathbf{n e g / 2 0 / 4 0 / 8 0 / 1 6 0}$ & & $136 / 6 / 5 / 0 / 0$ & $83 / 2 / 8 / 11 / 43$ & $91 / 4 / 24 / 23 / 5$ \\
Bizerte, $\mathbf{n}=\mathbf{1 1 8}$ & 91 & 0 & $2(7.4 \%)$ & $16(59.2 \%)$ \\
Distribution of VNT titres $\mathbf{n e g / 2 0 / 4 0 / 8 0 / 1 6 0}$ & & 0 & $25 / 0 / 0 / 1 / 1$ & $11 / 0 / 10 / 6 / 0$ \\
\hline
\end{tabular}


dog sera possessed TOSV-NT-Ab. Of the 11 sera that showed positive results with Toscana virus, 7 did not contain PUNV-NT-Ab demonstrating that these dogs had been infected with Toscana virus only and not with Punique virus. For the 4 remaining sera, the respective titres for TOSV-NT-Ab/PUNV-NT-Ab were 40/40 for one serum, 40/160 for one serum, and 20/160 for 2 sera. Three sera showed a difference $\geq$ two-fold dilutions in favour of Toscana virus, which is undisputable evidence of Toscana virus past infection. Therefore, past infection with Toscana virus is unambiguous for 10 of the 11 reactive sera. Since neutralisation is the most specific and discriminative serological technique; we can exclude that cross-reactivity is a valid explanation for the finding of NT Ab against two related viruses. Thus, it is most likely that these dogs have been successively infected by Toscana virus and Punique virus. Toscana virus is present in the governorate of Kairouan although it is much less frequently infecting dogs than Punique and Sicilian viruses.

In the governorate of Kairouan, the presence for TOSVNT-Ab in only $7 \%$ of dogs versus $43 \%$ of PUNV-NT-Ab was unexpected; indeed, it is in contrast with the high rate of TOSV-NT-Ab and low rate of PUNV-NT-Ab recently reported in humans from the governorate of Bizerte [5]. A possible explanation lies in differences of the phlebotomine fauna present in the two governorates. While $P$. perniciosus is the predominant sandfly species in the governorate of Bizerte, P. longicuspis is the most abundant sandfly species in the governorate of Kairouan $[2,10,12]$. Since both sandfly species are shown to be vector of phleboviruses [7], it is well conceivable that $P$. perniciosus is more anthropophilic compared to $P$. longicuspis which is more zoophilic leading to a difference in human versus dog biting rates and subsequently to a difference in the infection status with Toscana virus and Punique virus in humans compared to dogs within the governorates of Bizerte and Kairouan. To address this hypothesis, the forage ration of the two sandfly species needs to be determined in both governorates.

The high rate of SFSV-NT-Ab observed in the governorate of Kairouan demonstrates that Sicilian virus or a very closely virus related to Sicilian virus is present and circulates at high levels in the region. Such findings are in agreement with the identification of sequences corresponding to a Sicilian-like virus, provisionally named Utique virus in the village of El-Felta located within the governorate of Sidi Bouzid adjacent to the governorate of Kairouan [7].

The results obtained from the 27 sera collected from dogs in the governorate of Bizerte for which the VNT was interpretable showed that 16 sera contained neutralising antibodies against Sicilian virus which is coherent with the detection of Utique virus in 7 pools of sandflies collected from the same region in 2010 [7]. The results observed with Toscana virus (absence of positive serum), and Punique virus $(\mathrm{n}=2 ; 7.4 \%)$ cannot be extrapolated because of the low numbers, but confirms that Punique virus can infect not only humans [5] but also dogs.

The results of this study provided further evidence that Toscana, Punique and Sicilian viruses are present in Tunisia and showed that guard dogs may represent excellent sentinels for virus transmitted by sandflies. The existence and nature of vertebrate reservoir of sandflyborne phleboviruses is unknown; however, the high seroprevalence rates observed in dogs in this study leads to further investigations concerning the possible role of dogs in the transmission dynamic of theses arboviruses.

Whether Punique virus and Sicilian virus represent a threat for humans in Tunisia, as previously shown for Toscana virus [5], needs to be addressed in the future. For this, entomological studies combined with virological investigation should be organized as well as clinical studies in regional hospitals.

\section{Conclusions}

In conclusion, the results of this study showed that Toscana, Punique and Sicilian viruses are circulating in several regions of Tunisia, and dogs are frequently infected with these viruses for which they could serve as sentinels.

Competing interests

The authors declare that they have no competing interests.

\section{Authors' contributions}

All authors read and approved the final version of the manuscript.

\section{Acknowledgements}

This study was supported by the CRDF Global Grant N ${ }^{\circ}$ TNB1-31117-TU-13, the Pasteur Institute of Tunis, Tunis, Tunisia, and the Institute of Research for Development, Marseille, France. The research leading to these results has received funding from the European Union's Seventh Framework Programme for research, technological development and demonstration under Grant Agreement numbers 261504-EDENext-FP7 (http://www.edenext.eu) and FP7 CAPACITIES project GA no228292-EVA (European Virus Archive, http://www. european-virus-archive.com/). This article is catalogued by the EDENext Steering Committee as EDENext\#266. The work of RNC was done under the frame of EurNegVec COST Action TD1303.

\section{Author details}

Institut Pasteur de Tunis, Laboratory of Vector Ecology, Tunis, Tunisia. ${ }^{2}$ Aix Marseille Université, IRD French Institute of Research for Development, EHESP French School of Public Health, EPV UMR_D 190 "Emergence des Pathologies Virales", 13385 Marseille, France. ${ }^{3}$ HU Méditerranée Infection, APHM Public Hospitals of Marseille, 13385 Marseille, France.

Received: 24 September 2014 Accepted: 3 October 2014 Published online: 12 October 2014

\section{References}

1. Bahri O, Fazaa O, Ben Alaya-Bouafif N, Bouloy M, Triki H, Bouattour A: Role of Toscana virus in meningo-encephalitis in Tunisia. Pathol Biol (Paris) 2011, 59:e125-e127.

2. Bichaud L, Dachraoui K, Piorkowski G, Chelbi I, Moureau G, Cherni S, De Lamballerie X, Sakhria S, Charrel RN, Zhioua E: Toscana virus isolated from sandflies, Tunisia. Emerg Infect Dis 2013, 19:322-324. 
3. Es-Sette N, Nourlil J, Hamdi S, Mellouki F, Lemrani M: First detection of Toscana virus RNA from sand flies in the genus Phlebotomus (Diptera: Phlebotomidae) naturally infected in Morocco. J Med Entomol 2012, 49:1507-1509.

4. Es-Sette N, Ajaoud M, Bichaud L, Hamdi S, Mellouki F, Charrel RN, Lemrani M: Phlebotomus sergenti a common vector of Leishmania tropica and Toscana virus in Morocco. J Vector Borne Dis 2014, 51:86-90.

5. Sakhria S, Bichaud L, Mensi M, Salez N, Dachraoui K, Thirion L, Cherni S, Chelbi I, De Lamballerie X, Zhioua E, Charrel RN: Co-circulation of Toscana virus and Punique virus in northern Tunisia: a microneutralisation-based seroprevalence study. PLoS Negl Trop Dis 2013, 7:e2429.

6. Sghaier W, Bahri O, Kedous E, Fazaa O, Rezig D, Touzi H, Ben Yahia A, Meddeb Z, Triki H: Retrospective study of viral causes of central nervous system infections in Tunisia (2003-2009). Med Sante Trop 2012, 22:373-378.

7. Zhioua E, Moureau G, Chelbi I, Ninove L, Bichaud L, Derbali M, Champs M, Cherni S, Salez N, Cook S, de Lamballerie X, Charrel RN: Punique virus, a novel phlebovirus, related to sandfly fever Naples virus, isolated from sandflies collected in Tunisia. J Gen Virol 2010, 91:1275-1283.

8. Ben Ismail R: Incrimination de Phlebotomus perniciosus comme vecteur de Leishmania infantum. Arch Institut Pasteur Tunis 1993, 70:91-110.

9. Chargui N, Houas N, Slama D, Gorcii M, Jaouadi K, Essabah-Aguir N, Mezhoud H, Baba H: Transmission of visceral leishmaniasis in a previously non-endemic region of Tunisia: Detection of Leishmmania DNA in Phlebotomus perniciosus. J Vect Ecol 2012, 2012(38):1-5.

10. Zoghlami Z, Chouihi E, Barhoumi W, Dachraoui K, Massoudi N, Ben Hele K, Habboul Z, Hadhri MH, Limam S, Mhadhbi M, Gharbi M, Zhioua E: 2014. Interaction between canine and human visceral leishmaniases in a holoendemic focus of Central Tunisia. Acta Trop 2014, 139:32-38.

11. Cabezón O, Millán J, Gomis M, Dubey JP, Ferroglio E, Almería S: Kennel dogs as sentinels of Leishmania infantum, Toxoplasma gondii, and Neospora caninum in Majorca Island, Spain. Parasitol Res 2010, 107:1505-1508.

12. Zhioua E, Kaabi B, Chelbi l: Entomological investigations following the spread of visceral leishmaniasis in Tunisia. J Vect Ecol 2007, 32:371-374.

13. Nougairede A, Bichaud L, Thiberville SD, Ninove L, Zandotti C, de Lamballerie X, Brouqui P, Charrel RN: Isolation of Toscana virus from the cerebrospinal fluid of a man with meningitis in Marseille, France, 2010. Vector Borne Zoonotic Dis 2013, 13:685-688.

14. Karabatsos N: International Catalogue of Arboviruses Including Certain Other Viruses of Vertebrates. San Antonio, TX: Am Soc Trop Med Hyg; 1985.

doi:10.1186/s13071-014-0476-8

Cite this article as: Sakhria et al: Presence of sandfly-borne

phleboviruses of two antigenic complexes (Sandfly fever Naples virus and Sandfly fever Sicilian virus) in two different bio-geographical regions of Tunisia demonstrated by a microneutralisation-based seroprevalence study in dogs. Parasites \& Vectors 2014 7:476.

\section{Submit your next manuscript to BioMed Central and take full advantage of:}

- Convenient online submission

- Thorough peer review

- No space constraints or color figure charges

- Immediate publication on acceptance

- Inclusion in PubMed, CAS, Scopus and Google Scholar

- Research which is freely available for redistribution 